Service social

\title{
Quelques modèles d'analyse du processus d'élaboration des solutions morales chez les jeunes
}

\section{Françoise Digneffe}

Volume 40, numéro 1, 1991

Éthique et intervention sociale

URI : https://id.erudit.org/iderudit/706513ar

DOI : https://doi.org/10.7202/706513ar

Aller au sommaire du numéro

\section{Éditeur(s)}

École de service social de l'Université Laval

ISSN

1708-1734 (numérique)

Découvrir la revue

Citer cet article

Digneffe, F. (1991). Quelques modèles d'analyse du processus d'élaboration des solutions morales chez les jeunes. Service social, 40(1), 31-52.

https://doi.org/10.7202/706513ar
Résumé de l'article

Comment s'instituent les relations que chacun entretient avec les règles morales ? À cette question, les sociologues et les psychologues répondent en fonction de leur conception du processus de socialisation. Les uns, à la suite de Durkheim, ont tendance à définir celui-ci comme intériorisation de règles extérieures; les autres, suivant Piaget, comme formation d'une capacité d'« invention » de ces règles.

Dans une perspective interdisciplinaire et en s'appuyant sur des recherches relatives à la déviance et à la délinquance, cet article propose une conception du sujet comme acteur social, c'est-à-dire comme individu capable de définir les situations qu'il vit et de prendre distance par rapport à elles.

La relation que chaque acteur entretient avec les règles morales apparaît alors comme la mise en accord de ses projets, de ses désirs, du sens qu'il donne à sa vie avec les contraintes d'un monde « déjà là ». On parlera de délinquance comme gestion de sa vie à la manière dont Foucault a parlé de morale comme gestion de sa vie, c'est-à-dire de morale orientée vers l'éthique au lieu de morale orientée vers le code. 


\section{Quelques modèles d'analyse du processus d'élaboration des solutions morales chez les jeunes}

L'objectif de la recherche dont il est question dans le présent article ${ }^{1}$ consiste à comprendre ou à mettre en évidence le cadre de référence à partir duquel le sujet se situe vis-à-vis de ce qui, dans une société, est considéré comme " permis » ou comme "interdit ». II s'agit donc de rechercher et de s'interroger sur les différents éléments qui interviennent dans la manière dont le sujet se situe par rapport aux règles auxquelles il est confronté, en tenant compte de la situation et de la position qu'il occupe. Plus précisément, on envisagera tout d'abord les analyses de ce phénomène telles qu'elles apparaissent dans la tradition sociologique durkheimienne et centrées sur la socialisation, d'une part, et dans la tradition psychologique piagétienne et centrée sur l'apprentissage et l'interaction d'autre part. Une estimation des apports respectifs de ces théories nous conduira à proposer une perspective plus complexe qui tient compte non seulement des capacités de création du sujet, mais aussi du poids des institutions et des situations dans la recherche de solutions morales.

\section{Hétéronomie ou autonomie de la morale}

À propos de la socialisation et, plus particulièrement, de l'apprentissage ou de l'intériorisation des jugements moraux, on peut distinguer deux grands courants qui ont influencé le développement de la criminologie et qui ont été à l'origine d'un certain nombre de recherches. Notre objectif n'est pas ici de les opposer l'un à l'autre ou de les critiquer l'un par rapport à l'autre, mais de montrer dans quels contextes ils sont apparus ${ }^{2}$ et de mettre en évidence les résultats qu'ils sont susceptibles de produire ainsi que les enjeux qui les 
sous-tendent. Le premier courant, celui de la morale hétéronome, repose sur une conception durkheimienne de la socialisation comme intériorisation des règles et des modèles de comportement. II soustend plusieurs courants de recherche en criminologie portant sur la manière dont les jeunes se situent par rapport aux règles et aux valeurs. Le second courant repose sur une conception plus interactionniste de la socialisation. Il se fonde sur la conception piagétienne au terme de laquelle le sujet est considéré comme actif, capable dans une certaine mesure de créer les règles et de choisir les valeurs auxquelles il adhère. $C^{\prime}$ est dans ce courant que se situent les recherches effectuées par Kohlberg et ses collaborateurs (Kohlberg, 1958).

Il paraît d'autant plus intéressant de s'arrêter un moment sur le sens et la nature de ces deux courants de recherche que, dans le récent Dictionnaire critique de la sociologie, R. Boudon et Fr. Bourricaud les considèrent comme les deux modes de présentation de la socialisation qui constituent l'enjeu d'un débat fondamental au sein de la sociologie actuelle (Boudon et Bourricaud, 1983 : 485). Nous les envisagerons donc successivement avant de proposer une sorte de solution de remplacement à ces deux modèles.

\section{Le paradigme du conditionnement et la morale hétéronome}

Selon Piaget (1932), I'auteur de ce paradigme n'est autre que le " père » de la sociologie, Émile Durkheim, et il est lié à la conception que celui-ci se faisait de la société et, plus particulièrement, des rapports entre l'individu et la société. Dans une étude sur la théorie de la nature humaine chez Durkheim, Hawkins montre que " l'homme est décrit comme une créature plastique et malléable dont les comportements et les croyances, tout en ayant un substrat organique et psychologique, sont le produit du milieu social » (Hawkins, 1977). Et, de manière très schématique, on peut dire que, pour Durkheim, dans la mesure où la société (ou le groupe) transcende l'individu, dans la mesure où elle constitue un être sui generis qui possède une nature différente de la somme des individus qui la composent, elle leur impose ses propres valeurs qui sont à l'origine des règles de comportement. Dans La division du travail social (Durkheim, 1893), le sociologue français s'attache à montrer comment le sens des règles varie en fonction du degré d'évolution des sociétés et comment celles-ci sont instituées pour garantir la cohérence sociale et assurer un fonctionnement harmonieux des institutions. On comprend dès lors que, dans un tel système, les valeurs et les règles soient extérieures aux individus et que, dans L'éducation morale (Durkheim, 1925), Durkheim envisage la socialisation comme le pro- 
cédé par lequel les enfants, en grandissant, vont intérioriser les valeurs adoptées par les adultes. Pourtant, une analyse plus fine de cet ouvrage suscite le doute quant à la validité de cette thèse souvent avancée. II semble bien que la pensée de Durkheim soit plus complexe à cet égard, complexité liée par ailleurs à l'ensemble du système durkheimien.

Dans un premier temps, Durkheim expose ses idées sur ce qui constitue la base de l'éducation, à savoir la formation de l'esprit de discipline. Et, sur ce plan, sa conception de la morale comme un vaste système $d^{\prime}$ 'interdits est très claire : "l'ensemble des règles morales forme autour de chaque homme une sorte de barrière idéale au pied de laquelle le flot des passions humaines vient mourir " (Durkheim, 1925 : 91). L'éducation morale commence donc par l'apprentissage de l'esprit de discipline, de règles extérieures à l'individu et qui se transmettent de génération en génération. Ce n'est pas nous qui faisons la morale, dit Durkheim; et même si, en tant que membres de la société, nous apportons notre concours à l'élaboration de celle-ci, notre part reste très réduite. Notre attitude par rapport aux règles est beaucoup plus passive qu'active et il importe donc que l'enfant, dès son plus jeune âge, apprenne cette attitude, qu'elle lui devienne en quelque sorte naturelle. Grâce à l'autorité du maître et des parents, intermédiaires entre lui et la société, l'enfant sentira qu'il y a au-dessus de lui un pouvoir qui le dépasse et auquel il doit obéir. Ainsi, l'apprentissage de l'esprit de discipline correspond à l'exercice de la morale du devoir et se caractérise par l'esprit de sacrifice, l'effort, la contrainte, la lutte contre soi-même pour se conformer à la règle.

Voilà sans doute les pages les plus connues de Durkheim à propos de l'éducation, celles auxquelles on se réfère le plus souvent et qui ont d'ailleurs servi de base à l'élaboration de toute la pédagogie traditionnelle. II s'agit, comme on le voit, d'une vision légaliste de la morale, qui se caractérise par la nécessité de la soumission face à un système d'interdits qui dépasse l'homme. C'est également une vision fondée sur une conception inégalitaire des rapports entre enfant et adulte, entre élève et maître où les aînés apparaissent comme transmetteurs d'un savoir moral qu'il s'agit donc d'imposer sans discussion. C'est elle que l'on qualifie de vision hétéronomique de la morale.

Mais l'esprit de discipline ne constitue qu'un aspect, une face de la morale durkheimienne, il exprime " ce qu'il y a de plus formel dans la vie morale " (Durkheim, $1925: 47)$. Quelque chose d'autre va devoir remplir cette forme vide, lui donner un sens, car la régulation en elle-même, si elle n'est pas sous-tendue par une fin, n'a pas de sens.

Cette fin, l'individu la trouvera dans sa participation positive au groupe, qu'il s'agisse de " la famille, la patrie, l'humanité » (Durk- 
heim, $1925: 51$ ). II la trouvera dans son attachement à un autre que lui-même, mais qui ne soit pas un simple individu qui est en réalité pareil à lui-même. II faut qu'il s'attache à un être qui dépasse l'individu et vers lequel il se sente attiré, qui deviendra alors le ferment de la cohésion et de la solidarité sociales. Ainsi, on pourra dire que la fin de la morale se situe au-delà de l'homme et que le groupe, ou plus exactement la société, prend, dans la perspective durkheimienne, la place du Dieu de la religion chrétienne. C'est l'attachement au groupe qui fondera désormais le respect de la règle. Mais celui-ci ne résultera plus de la discipline passive, mais il sera sous-tendu par les "forces actives et inventives de la conscience " (Durkheim, 1925 : 87). La théorie de l'éducation ne se centre dès lors plus sur le rapport à l'autorité, mais sur l'importance qu'il y a à susciter chez les jeunes des motivations à agir, à éveiller leur imagination et leur créativité. "II faut entraîner les individus à la poursuite de grandes fins collectives auxquelles ils puissent s'attacher; il faut leur faire aimer un idéal social à la réalisation duquel ils puissent travailler un jour » (Durkheim, 1925 : 87). L'intégration au groupe prend donc une place aussi importante que la soumission, l'activité positive de participation à une tâche commune prime sur le respect de la morale établie. L'hétéronomie de la morale pourrait alors être remise en question. Pourtant, qu'il s'agisse de se soumettre à des règles extérieures à l'individu ou d'agir par attachement aux groupes, par devoir ou par inclination, la fin de l'acte moral reste la société, envisagée comme entité transcendante et distincte des individus concrets et particuliers qui la composent. Et les enfants, dans une telle perspective, sont considérés comme des êtres incomplets qui ont tout à apprendre des adultes vus comme des courroies de transmission entre la société ou la conscience collective et les consciences individuelles.

À partir d'une telle conception, on pensera naturellement que, dans une société donnée, si l'on en détermine le système de valeurs, on pourra étudier la manière dont les individus se situent par rapport à celui-ci et mesurer ainsi l'efficacité des mécanismes d'intériorisation que l'on peut observer. Ainsi en sera-t-il des effets de la punition, de la récompense, des relations affectives entre parents et enfants, etc. On peut dire que c'est à partir d'une telle représentation que se sont développées les recherches qui, en psychologie sociale, reposent sur la théorie du conditionnement opérant (Debuyst, 1985). On constate également que, dans le domaine de la criminologie, ce modèle a inspiré plusieurs courants de recherche comme les théories relatives à la formation des sous-cultures déviantes ou délinquantes. Celles-ci prétendent en effet que, dans une société donnée, les individus qui ne peuvent obtenir de satisfactions suffisantes en respectant les règles de la société dominante vont se regrouper et, par un méca- 
nisme de formation réactionnelle, constituer des sous-groupes dans lesquels apparaissent des valeurs et des modes de comportement propres. Là aussi, on peut dire que c'est le sous-groupe, considéré comme une entité, qui domine les individus et leur impose ses propres normes (Cohen, 1955). Un tel modèle inspire également les recherches effectuées dans la perspective de Hirschi (1969) et sa théorie du lien social et dont on connaît le formidable développement aujourd'hui.

Ce qui unit tous ces courants de recherche pourtant bien différents les uns des autres $c^{\prime}$ est, nous semble-t-il, le caractère non problématique de la règle qui est considérée comme un donné. La vie morale et sociale s'organise autour de l'idée d'une règle extérieure et qui s'impose. Le sujet se perçoit comme respectant ou intégrant une règle qui va de soi et qui est donnée comme évidente. Cette perspective va de pair avec un certain relativisme parce que, pour le sujet, l'entité extérieure apparaissant comme créatrice de la règle est la collectivité à laquelle il appartient et que cette collectivité possède des caractéristiques spécifiques, qu'elle peut être divisée en sousgroupes, qu'elle peut changer dans le temps. II n'en reste pas moins que c'est le groupe qui institue les règles nouvelles.

Dans un tel cadre, la morale s'articule autour de l'extériorité de la règle et d'une certaine relativité de celle-ci. Pourtant, chez Durkheim lui-même, un tel modèle ne peut être entièrement adéquat. En effet, une attention parfaite de chacun à toutes les règles extérieures et une observation absolue de celles-ci est inconcevable, car elle entraînerait la paralysie de la société elle-même ou, plus exactement, l'impossibilité de tout changement social. C'est pourquoi, pour Durkheim, la délinquance est normale dans une société et elle est de surcroît utile.

\section{Le paradigme de l'interaction et la morale autonome}

Dans Le jugement moral chez l'enfant, Piaget (1932) introduit sa recherche sur le développement du jugement moral en s'opposant directement à la thèse durkheimienne. II propose un nouveau paradigme centré, non plus sur la société comme productrice de règles, mais sur les relations interindividuelles qui se produisent dans la société. Dès lors, la construction théorique qui en résulte sera fondamentalement différente de celle du sociologue français. En effet, reconnaissant jusqu'à un certain point le bien-fondé des analyses de L'éducation morale, Piaget leur adresse néanmoins une critique fondamentale qui résulte du fait qu'elles n'envisagent qu'un aspect de la morale enfantine : en effet, cet ouvrage est situé dans une perspective pédagogique selon laquelle l'apprentissage des règles et des valeurs ne peut avoir lieu que dans le cadre des relations que l'enfant 
entretient avec les adultes, parents ou maîtres. Ce faisant, dit Piaget, Durkheim omet la spécificité et le rôle des relations entre enfants, c'est-à-dire entre pairs et égaux. Or, il s'agit là d'un élément capital dans la formation de la conscience morale et dans l'explication de la manière dont les règles s'élaborent et sont vécues. Si le processus de socialisation s'effectue en partie par les relations entre adultes et enfants, entre le pouvoir des institutions et les sujets qui s'y adaptent, l'apprentissage proprement moral est, selon Piaget, à mettre en relation avec ce qui se passe entre les enfants, avec la manière dont ils apprennent à vivre ensemble, à se comprendre, à s'accepter et, de ce fait, à se constituer leurs propres normes de conduite.

On sait que, pour Piaget, l'enfant n'est pas seulement un adulte en devenir, qu'il n'est pas un être incomplet, mais qu'il constitue un sujet à part entière, avec ses capacités propres de compréhension et sa vision du monde originale. On trouve pour l'apprentissage moral ce que Piaget affirme également pour l'apprentissage en général, c'est-à-dire l'importance de l'interaction entre le sujet et le milieu, le rôle actif que l'enfant joue dans sa propre évolution et également le fait que, à chaque stade de son développement, il ne s'agit pas pour lui de se perfectionner mais de se transformer, de passer d'une vision du monde à une autre, chacune possédant sa cohérence et sa logique propres.

L'intérêt de Piaget va alors se tourner vers ce moment où l'enfant passe d'une morale hétéronome, centrée sur une obéissance aux règles imposées par les adultes, à une autre morale qu'il appelle autonome. Pour Piaget, on peut dire que, déjà dans le cas de la morale hétéronome, c'est le rapport à la personne qui donne un sens à la règle, par opposition à Durkheim pour qui les personnes ne sont que des intermédiaires entre l'enfant et la société productrice de règles. On assiste donc, d'un point de vue théorique, à une sorte de désacralisation de la règle, parallèle à une conception de la société formée d'abord par les relations entre individus et non pas transcendante à ceux-ci. La transformation du vécu moral de l'enfant pourra s'opérer quand celui-ci se rendra compte que, lorsqu'il joue avec ses camarades, le respect qu'il a pour la règle ne résulte pas du fait que ce sont des adultes qui lui ont expliqué comment il fallait jouer, mais que $c^{\prime}$ est à travers ses relations avec les enfants qui jouent tel ou tel jeu que doivent s'établir des consensus pour que le jeu soit possible. Vient un moment où ce qui importe ce n'est plus d'obéir à la consigne parce qu'elle aurait un caractère sacré dans la mesure où elle émane de l'adulte, mais de se mettre d'accord, entre soi, sur les règles qu'on décide ensemble de respecter. "La consigne s'efface pour devenir accord mutuel et les règles, devenant librement consenties, perdent leur caractère d'obligation externe »(Piaget, $1932: 309$ ). 
Considérant le monde du jeu comme une micro-société, Piaget va commencer par observer comment, aux différents âges, les enfants se situent par rapport aux règles du jeu. Puis il dialogue avec eux, les soumettant à des dilemmes moraux, pour comprendre comment, toujours en fonction de leur âge, ils donnent une signification particulière qui les amène à adopter telle ou telle solution. Ainsi il parvient à mettre en évidence l'existence de stades du développement moral.

La première phase est dite de "réalisme moral ». À ce stade, les règles imposées par les adultes constituent, avant même d'être assimilées par l'esprit, des obligations catégoriques. Elles acquièrent la valeur de nécessités rituelles et les choses défendues prennent la signification de tabous. Le bien n'est défini alors que par l'obéissance et la règle doit être observée à la lettre, plutôt que dans son esprit : "la contrainte adulte produit une sorte de réalisme de la lettre" (Piaget, 1932 : 309).

Dans une deuxième phase, intermédiaire, l'enfant n'obéit plus seulement aux ordres de l'adulte, mais à la règle elle-même, généralisée et appliquée indépendamment de la contrainte adulte. Le sujet se trouve alors dans un état de demi-autonomie : il y a toujours une règle qui vient du dehors, mais le travail de l'intelligence permet de combiner et de généraliser les différentes règles qui viennent de l'adulte. Dans cette phase, l'égalité prime sur l'autorité avant de faire place à une recherche de l'équité qui n'est plus objective, mais tient compte des points de vue et des situations respectives de chacun. Concrètement, cette évolution est favorisée par la diminution de la surveillance exercée par les adultes sur les enfants et par le fait que l'enfant se sent de moins en moins dominé par ses aînés.

Enfin, dans une troisième phase, la règle est véritablement désacralisée et son acceptation et son observation seront liées au consensus dont elle a pu faire l'objet. C'est d'un accord entre des pairs que la règle va émaner; ce sont eux qui, d'une certaine manière, la réinventent ou la créent. La relation au sein de laquelle cette création est possible se caractérise par la coopération entre les membres d'un groupe ou d'une société et par le respect mutuel dont chacun fait preuve à l'égard des autres.

$\mathrm{Ici}$, une précision s'impose et oblige à situer l'assentiment aux règles morales par rapport au respect des lois. Pour Piaget, l'avènement des démocraties correspond dans une certaine mesure à une évolution de l'humanité qui suit les mêmes étapes de développement que chaque individu. Les gouvernements autoritaires des sociétés primitives sont peu à peu remplacés par les régimes consensuels et démocratiques des sociétés développées. Néanmoins, Piaget reconnaît que les lois dans une démocratie possèdent un statut différent de 
celui des règles morales (Piaget, 1965). Elles revêtent en effet un caractère d'universalité et d'impersonnalité que n'ont pas les règles morales dont le respect est lié et sous-tendu par le respect pour autrui. Si l'on parle de respect des obligations légales, c'est dû, semble-t-il, à une extension des significations de ce concept, car il s'agit en réalité d'un autre sentiment que Piaget appelle le sentiment de reconnaissance (Piaget, 1965 : 190). Le respect est, on s'en souvient, un sentiment qui naît entre deux individus et exprime la valeur attribuée à l'autre. La reconnaissance d'un droit ou d'une loi est un " sentiment impersonnel qui n'évalue pas une personne, mais une 'fonction' ou un 'service', c'est-à-dire un aspect particulier de la personne " (Piaget, 1965 : 192). Même si les lois, au moment où elles sont promulguées, résultent $d^{\prime} u n$ consensus entre des individus, une fois édictées elles s'inscrivent dans un rapport juridique qui suppose une simple conservation des valeurs acquises, tandis que les rapports moraux restent toujours des rapports personnalisés où chacun s'efforce de s'ouvrir au point de vue de l'autre.

L'étude du développement du jugement moral chez l'enfant, c'est donc l'étude du passage de l'égocentrisme à l'altruisme, c'est-àdire à la reconnaissance et au respect du point de vue propre de l'autre et à une vision plus relativiste de la règle morale. C'est également la mise en évidence d'une conception du sujet entendu comme créateur, acteur et inventeur de règles nouvelles. "Comprendre c'est inventer ", disait Piaget et cela est d'autant plus vrai si l'on se place dans le domaine moral.

\section{L'évolution des recherches réalisées dans la perspective piagétienne}

\section{Bilan des recherches}

On sait que Piaget lui-même n'avait pas accordé une importance très grande à sa recherche sur le développement du jugement moral et ne poursuivit pas cette piste de travail. Celle-ci fut réactualisée quelque trente années plus tard par un psychologue américain, L. Kohlberg, qui prolongea donc ces recherches ${ }^{3}$.

Kohlberg, à la suite de Piaget, insiste sur le fait que l'enfant n'est pas un adulte en puissance, mais qu'aux différents âges il existe une manière propre de comprendre l'ordre socio-moral. Le développement se définit alors en termes de réorganisation qualitative des structures de pensée et non plus en termes d'apprentissage. Dans un tel cadre, la maturation morale se caractérise par la possibilité progressive de tenir compte des intentions et du point de vue d'autrui dans la 
manière d'apprécier la valeur d'un acte. C'est ainsi que Kohlberg définit les trois niveaux de développement : pré-conventionnel ou égocentrique; conventionnel, c'est-à-dire qui tient compte du point de vue des autres et de leurs attentes; enfin, post-conventionnel, où la perspective est centrée sur des principes moraux abstraits qui peuvent entrer en conflit avec les valeurs de la société conventionnelle. À ce niveau, le sujet adhère à un système de valeurs considérées comme universelles et dont le fondement ultime est la justice.

Le modèle proposé par Kohlberg a alors permis le développement de nombreuses recherches en criminologie. Depuis longtemps, en effet, les chercheurs s'étaient posé la question de savoir quelles étaient les valeurs sociales et morales auxquelles adhéraient les jeunes délinquants et pourquoi. Ils s'étaient également penchés sur l'étude des mécanismes d'intériorisation de ces valeurs. Mais, la plupart du temps, ces recherches étaient fondées sur des théories envisageant la socialisation sur le mode des relations stimulus-réponse, c'est-à-dire que le sujet est conditionné à réagir d'une certaine manière à tel ou tel type de stimulus (Debuyst, 1985). On se situe alors sur le plan du paradigme du conditionnement.

L'approche structuro-développementale permet de poser les problèmes d'une manière radicalement différente. Plutôt que de se centrer sur le contenu de l'orientation morale des délinquants, elle va étudier la structure du processus d'évolution morale. Son intérêt va s'étendre au-delà des valeurs auxquelles les jeunes souscrivent pour chercher à comprendre comment - en tant qu'interprètes actifs de leur propre expérience - ils conceptualisent les valeurs et les problèmes de nature morale.

De nombreuses recherches comparatives entre délinquants èt non-délinquants ont été réalisées. Les premières études menées par Kohlberg en 1958 tendaient déjà à mettre en évidence le fait que les jeunes délinquants raisonnaient au niveau pré-conventionnel, tandis que le groupe contrôle de non-délinquants se situait au niveau conventionnel. Mais, quinze ans plus tard, un bilan de toutes les études réalisées (Jurkovic et Prentice, 1980) montre notamment que si certains délinquants raisonnent à un niveau plus bas que les non-délinquants, certains autres utilisent des stades moraux plus élevés que les non-délinquants. II montre également que le niveau de jugement moral est plus élevé lorsque les dilemmes utilisés renvoient à des situations plus concrètes et surtout plus familières aux jeunes des classes inférieures. Enfin, il ne parvient pas à établir de différence significative entre le niveau de développement moral des familles de jeunes délinquants et celui des autres.

Ces recherches comparatives ont été effectuées en tenant compte de variables telles que le statut économique, l'âge, le sexe, 
la race et l'intelligence. Mais les variables liées au statut même des enfants, relatives au fait d'avoir été appréhendé, jugé, au fait d'être détenu ou d'être en institution n'ont été que très rarement retenues. On peut dès lors se poser la question de savoir si de telles recherches ne mesurent pas les effets de la réaction sociale sur le jugement moral plutôt que ceux de la délinquance. De toute façon, elles ne laissent apparaître aucune différence significative entre les différents groupes d'enfants.

\section{Morale de justice et morale de responsabilité}

Après avoir travaillé durant plusieurs années avec Kohlberg et avoir écrit avec lui un important article théorique intitulé The Adolescent as a Philosopher (1971), Carol Gilligan a pris ses distances en raison d'un certain nombre de motifs d'ordre théorique qu'elle a élaborés à partir des résultats des nombreuses recherches empiriques qu'elle avait réalisées en suivant la méthode de Kohlberg4.

De manière plus précise, $C$. Gilligan a effectué plusieurs recherches sur le développement du jugement moral des femmes qui l'amenèrent à constater que, dans la plupart des cas, les résultats indiquaient que les femmes disposaient d'un niveau de jugement moral inférieur à celui des hommes. Réfléchissant à partir de ces résultats, elle observe que les théories à propos du développement du jugement moral se fondent sur une conception de l'individu prédominante en Occident et qui n'a été remise en question par aucun des psychologues qui ont travaillé sur cette question, tels Piaget, Erikson ou Kohlberg. Cette conception part d'un a priori selon lequel l'individu, en grandissant, acquiert son identité en se différenciant de plus en plus des autres sujets et en apprenant à penser d'une manière rationnelle qui le libère progressivement du poids des événements concrets. En d'autres termes, il pense d'une manière de plus en plus formelle, passant de la théorie au fait, du général au particulier, « il se définit lui-même et définit la morale par l'autonomie, en opposition avec les conventions qui font la particularité du monde de l'enfance " (Gilligan, 1977).

Or, on peut dire qu'il ne s'agit pas là d'une construction scientifique, mais d'une conception qui correspond aux stéréotypes de l'âge adulte masculin et qui apparaissent tels quels dans un grand nombre de recherches scientifiques. Ces stéréotypes ne correspondent naturellement pas à ceux des femmes qui, étant donné leur position sociale et culturelle, sont amenées, en ce qui concerne le développement du jugement moral, à tenir compte, de manière de plus en plus fine et complexe, du contexte dans lequel les dilemmes moraux viennent prendre place. Cette manière propre aux femmes de 
se situer, au lieu d'être envisagée comme un problème et d'être prise en compte par les chercheurs, était résolue en disant simplement que les femmes avaient un niveau de développement moral inférieur aux hommes, ou encore qu'elles manifestaient une sorte de "déviance " ou de "déficience morale " (Gilligan, $1977: 482$ ).

C'est pourquoi C. Gilligan a décidé d'envisager l'analyse de cette différence et de sa signification par rapport à la définition de la sphère de la moralité comme un point central de sa recherche. Elle met en évidence le fait que, dans la perspective piagétienne, le jugement moral a été considéré, à l'image du processus purement cognitif, comme un processus tendant vers la recherche et l'adoption de solutions rationnelles, comme s'il existait, dans le monde social, des solutions rationnelles à des situations conflictuelles dans lesquelles, à un certain point de vue, le fait même de choisir " semble faire violence à la justice » (Gilligan, $1977: 483$ ). Cette conception rationnelle du développement du jugement moral semble globalement confirmée en ce qui concerne les garçons. Mais elle aboutit, comme on l'a $\mathrm{vu}$, à considérer les femmes comme " déviantes " par rapport à ce modèle. Comparant de manière plus systématique la façon dont les hommes et les femmes se situent par rapport aux problèmes moraux (Gilligan, 1982), elle découvre alors que, en réalité, les dilemmes moraux de Kohlberg, construits à partir d'une conception de la morale en termes de justice, de droit et d'équité, ne sont pas susceptibles de prendre en compte l'orientation morale des femmes. Celles-ci seraient beaucoup plus influencées par le "souci » d'autrui (care) et la responsabilité vis-à-vis des conséquences d'un acte posé, tandis que l'orientation morale des hommes serait plus orientée vers la justice et l'équité. La conception "féminine » de la morale consisterait fondamentalement dans la recherche de solutions à des conflits opposant non des droits, mais des responsabilités vis-à-vis des conséquences possibles d'une action ou d'une décision. Cette morale de responsabilité ne remplace pas la morale de justice, mais elle constitue une approche différente des problèmes moraux, une approche qui repose sans doute sur une conception moins " rationnelle » de l'être humain. Le sujet responsable, c'est nécessairement un sujet en situation, en relation avec les autres, qui n'applique pas des principes, mais qui tente de résoudre les problèmes en tenant compte non des droits de chacun mais en fonction d'une attention à la personne concrète qui est concernée.

Poursuivant dans la ligne de recherche tracée par C. Gilligan, on aboutit à une conception de la morale comme le lieu où se prennent des choix qui sont chaque fois "uniques", où les problèmes se résolvent par une prise de responsabilité, mais surtout à partir d'un mode de pensée qui est contextuel et inductif plutôt que formel et 
abstrait. En d'autres termes, le concept de personne n'y est plus perçu de manière abstraite et isolée, mais il est envisagé dans ses relations avec les autres, en fonction des types de situations dans lesquelles on se trouve.

En conclusion de ceci il apparaît que, dès que le sociologue ou le psychologue tente d'une manière ou d'une autre de "mesurer " le développement du jugement moral, il fait face au problème philosophique de la définition de la morale ou de l'éthique. Il est obligé de s'engager, de choisir une définition qui ne peut être seulement conventionnelle, mais qui doit refléter la manière dont il se représente les processus d'évaluation par rapport à ce qui peut ou doit être considéré comme bien ou mal, à faire ou à ne pas faire. Ainsi, on voit bien que ce qui oppose Kohlberg à Gilligan, ce n'est pas un simple désaccord dans l'interprétation des résultats, mais c'est une autre manière de définir la moralité. Et en ce qui concerne notre problématique propre, il apparaît que la définition proposée par Gilligan correspond mieux à une approche phénoménologique et situationnelle du sujet.

On définira alors la sphère de la moralité ou le domaine de l'éthique comme la sphère de la vie du sujet qui est concernée par les évaluations et les choix qu'il fait compte tenu des conséquences de ces choix pour lui (au niveau d'une certaine forme de réalisation de soi) et pour les autres. Ainsi, l'apprentissage " moral "serait différent de l'apprentissage social en ce qu'il ne serait pas, ou pas seulement, une recherche d'adaptation au monde où vit le sujet. II procéderait, d'une part, d'une sorte de réflexion liée à un projet (implicite ou explicite) de réalisation de soi comme sujet, et à une certaine gestion de sa vie au regard de ces projets; et d'autre part, à une prise en compte ou à une reconnaissance des projets d'autrui qui peuvent venir interférer avec les siens propres. Une telle vision de l'apprentissage moral se rattache à une conception de l'éthique comme "visée de l'universel ", c'est-à-dire, selon le philosophe J. Ladrière, que cette visée, tout en comportant une exigence de portée universelle, "reste affectée $d^{\prime}$ 'une certaine incertitude, et (qu') elle est de toute façon sous-déterminée par rapport aux exigences concrètes de l'action » (Ladrière, 1984 : 39).

Il apparaît donc que, dans une situation donnée, la manière dont le sujet organise son rapport aux règles relève de processus complexes. L'interaction qui se produit entre le sujet et les normes dépend à la fois de I'histoire personnelle du sujet, c'est-à-dire du système de relations dans lequel il vit, et de sa position au sein de la structure sociale. Le monde moral qu'il se construit émane lui-même d'une "vision du monde " comme horizon de sens et il peut être jusqu'à un certain point considéré comme le reflet de l'idéologie; mais il n'est pas que cela, car il contient également une possibilité 
de prise de distance, de prise de responsabilité. En d'autres termes, le sujet est déterminé par un certain nombre d'éléments situationnels tout en étant acteur de son histoire. Mais une telle conception requiert d'autres méthodes d'approche que celles de Piaget et de Kohlberg.

\section{Acteur social et sujet moral}

Détermination en dernière instance du social chez Durkheim, mise à l'avant-plan des interactions individuelles chez Piaget et ses successeurs, tels sont sans doute les éléments essentiels à retenir du parcours réalisé jusqu'à présent. $D^{\prime} u n$ côté, les règles morales tendent à s'imposer de manière non problématique et leur intériorisation plus ou moins adéquate dépendra de la position des individus dans la société ou de leur appartenance à des groupes spécifiques; d'un autre côté, le primat donné à l'interaction fait passer à l'arrière-plan le poids des institutions, donnant à penser que le jugement moral pourrait se former de manière indépendante, autonome, et qu'il serait essentiellement lié au développement des structures cognitives.

C. Gilligan, insistant sur un aspect central du jugement moral, à savoir la prise en compte des conséquences des actes en rapport avec le souci des autres (et le souci de soi), introduit une dimension plus "sociale » dans le développement du jugement moral : celui-ci devrait être étudié en tenant compte du contexte dans lequel il est produit, car c'est au sein de celui-ci qu'il vient à prendre son sens. Ce faisant, elle inscrit en quelque sorte le sujet dans le social, elle montre comment des structures affectives et cognitives se forment et déterminent le jugement dans des situations concrètes. Pourtant, cette dimension n'est pas approfondie chez elle et reste dominée par une perspective développementale qui isole malgré tout le sujet du social, qui en tout cas reste centré sur une perspective micro-sociologique. Pour avancer un peu plus loin dans la voie qui paraît ouverte, nous voudrions proposer quelques aspects d'une perception du sujet comme acteur social en indiquant les effets qu'il peuvent avoir pour une nouvelle conception de l'organisation du rapport à la morale ou à l'éthiques.

En criminologie, le fait d'adopter le paradigme de l'acteur social vise à sortir des fictions du libre arbitre et du déterminisme telles qu'elles ont été imposées par les tenants de l'école pénale classique $d^{\prime}$ une part et les positivistes italiens $d^{\prime}$ autre part ${ }^{6}$. Pour les premiers, l'idée d'un sujet doué de libre arbitre permettait de le considérer comme responsable de ses actes, tandis que la fiction d'un être dont le comportement est déterminé par des facteurs amenait plutôt les 
seconds à voir le droit pénal comme instrument de défense sociale (Debuyst, 1990). Par rapport à ces perspectives, l'acteur social apparaît comme un sujet " doté d'un point de vue propre qui dépend de la position qu'il occupe dans le cadre social, de l'histoire qui a été la sienne et des projets autour desquels son activité s'organise (Ibid. : 26).

\section{Acteur social et sociologie de l'action?}

Cette notion d'acteur social trouve son origine dans les travaux de Touraine et surtout dans ses études publiées dans Le retour de l'acteur (1984). Elles apparaissent en effet comme une manière originale de se situer par rapport à la question de la socialisation et de proposer une voie qui, nous semble-t-il, est susceptible d'ouvrir des perspectives nouvelles dans le cadre de la problématique qui nous occupe.

Plutôt que d'envisager la socialisation comme un processus unique, on la situera par rapport aux caractéristiques générales de l'action sociale qui, dans la perspective tourainienne, supposent une distinction entre trois logiques ou trois niveaux fondamentaux (Dubet, 1990).

a) Le premier niveau est celui de l'intégration. La société y est conçue comme une organisation et la socialisation des acteurs vise l'intériorisation des rôles, des normes et des valeurs correspondant à des statuts et à des positions. C'est très globalement la perspective inaugurée par Durkheim et reprise par les fonctionnalistes. On pourrait également dire qu'elle correspond à une manière de considérer la société comme un ensemble d'institutions déjà "instituées ", qu'elle insiste sur cet aspect et se centre dès lors sur l'étude des mécanismes d'héritage et de transmission des valeurs, des goûts, des opinions.

b) Mais, on l'a vu, la socialisation ne peut être réduite à l'apprentissage d'un code rigide, elle s'effectue à travers des relations, à travers des interactions et $c^{\prime}$ est la raison pour laquelle Piaget avait considéré le jeu entre les pairs et, plus largement, les relations entre pairs comme la source $d^{\prime}$ une "loi morale plus abstraite et plus générale que celle qui découle de la simple identification à l'autorité parentale " (Dubet, $1990: 67$ ). Dans cette perspective, ce n'est plus l'imitation des anciens ou des supérieurs qui commande le jeu mais c'est une règle de compétition plus démocratique et plus égalitaire. Ce qui, dans la conception piagétienne, consistait dans l'apprentissage d'une morale autonome est introduit ici d'une manière plus complexe, dans le vocabulaire de G.H. Mead, comme l'existence, au sein du sujet, d'une instance autonome ou Je à côté du Moi. Le Je représente le facteur de créativité, de spontanéité, d'imprévisibilité 
dans le comportement humain parallèlement au Moi qui se construit par intériorisation progressive des rôles sociaux. Mais ici les deux instances (Je et Moi) sont le produit des échanges sociaux et non plus seulement des interactions interindividuelles. Cela sera interprété par la sociologie des organisations comme la capacité qu'ont les acteurs de construire des stratégies en vue de réaliser leurs intérêts. L'autonomie du sujet moral ne peut s'inscrire ici dans un projet social, elle s'inscrit dans la défense d'intérêts individuels ou de groupe, " conformément à des stratégies tant défensives qu'offensives " (Touraine, 1984 : 93). Perçue de cette manière, la conception piagétienne, trop abstraite, ne peut être envisagée que de manière réductrice.

c) Vient alors le niveau de la socialisation propre à la sociologie de l'action. Celle-ci considère que la société ne peut être définie comme la seule juxtaposition d'un système d'intégration et d'un système formé de l'ensemble des lieux stratégiques d'interaction, elle comporte aussi ce que Touraine désigne par l'historicité. Une société historique construit l'image de l'action humaine et des valeurs qui permet aux acteurs, non seulement de donner un sens à leur action, mais aussi de s'en distancer et de la juger. En d'autres termes, dans les sociétés historiques, "l'action a un sens pour l'acteur, non seulement parce qu'elle vise des buts et parce qu'elle s'inscrit dans un contexte culturel, mais aussi parce que l'acteur peut s'en distancer, s'en expliquer et en rendre compte "(Dubet, 1990 : 64). Cette affirmation signifie donc que l'acteur est capable de réfléchir sur luimême, qu'il est un sujet. Mais aussi un sujet de droit qui a à se situer par rapport à des normes juridiques, $d^{\prime}$ une part, par rapport à un univers éthico-moral d'autre part. Et ces univers représentent un registre spécifiquement anthropologique mais sont cependant différents l'un de l'autre." Les animaux ont des régulations naturelles, seuls les hommes se constituent des normes qui ont le même caractère de 'vide contredit' que la notion de personne ou de sujet. II y a une différence entre cet univers de la norme (qui est, tout de même, celui du crime, du droit, etc.) et celui de l'univers des personnes auquel renvoient le terme de social et peut-être aussi celui d'acteur... La norme est un phénomène aussi radicalement anthropologique que la personne, mais ils sont différents" (Schotte, 1990 : 139).

Ainsi, la sociologie de l'acteur social est-elle à même de prendre en compte, d'intégrer une vision du sujet nettement plus complexe que celle des sociologies antérieures. Pour elle, "le sujet se saisit, au-delà de ses œuvres et contre elles, comme silence, comme étrangeté au monde dit social et aussi comme désir de rencontre avec l'autre, reconnu comme sujet " (Touraine, 1984 : 15). Pour A. Touraine, le sujet n'est pas opposé à l'acteur, il est porteur de significations qui sont susceptibles de s'exprimer de manières diverses, mais 
qui toutes doivent être prises en considération. On peut dire que le sujet-acteur est aussi capable de prises de distance que d'engagements, selon les valeurs qu'il accordera à l'un ou à l'autre et selon les réseaux de relations dans lesquels il est placé. Le sujet et l'acteur sont alors les "deux faces de l'identité » (Touraine, 1984 : 165). L'une apparaît comme un refus des rôles sociaux et comme un refus de la définition sociale des rôles que doit jouer l'acteur; c'est un appel, contre les rôles sociaux, à la vie, à la liberté, à la créativité. Mais cette perspective défensive, cet appel à une identité de moins en moins sociale, se double, dans les sociétés développées, de revendications de plus en plus directement politiques, et qui s'expriment alors, non sur le plan de l'identité, mais des rapports sociaux et des pouvoirs (Ibid. : 172).

Ainsi, cette quête d'identité inscrit le sujet-acteur dans un système de relations. G.H. Mead et Piaget avaient déjà montré le caractère essentiel des interactions dans la formation et le développement d'un sujet. Mais Touraine va plus loin. Si l'on se contente d'étudier les interactions, on manque l'essentiel pour une compréhension de celles-ci, c'est la connaissance du " champ de relation " (Ibid. : 111) qui s'inscrit toujours dans un rapport de pouvoir. Et, poursuit Touraine, pour analyser ce champ, il nous faut complètement renverser notre vision spontanée de l'organisation sociale. Car, pour celle-ci, "les règles, les normes, semblent préexister, comme un décor de théâtre, au moment où les acteurs entrent en scène ". Alors qu'en réalité, la règle n'est pas antérieure à l'acte, " elle est produite, modifiée et contestée par chaque acte. L'ordre n'est ni intangible, ni cohérent. II n'est qu'une mise en forme partielle des rapports sociaux, des transformations culturelles et des conflits de pouvoir et d'autorité » (Ibid. : 113).

Étudier les acteurs dans leurs champs de relation amène donc à considérer les règles et les normes comme la résultante de rapports entre acteurs sociaux qui négocient ou s'affrontent à partir de positions de pouvoir différentes. Et les acteurs vivent, agissent, respectent ou transgressent les règles, jouent un rôle qui renforce ou au contraire ébranle ou remet en question des règles imposées un moment ${ }^{8}$.

\section{Acteur social et éthique. Introduction à une approche biographique}

Si une telle conception paraît aujourd'hui fructueuse pour une analyse du fonctionnement des sociétés occidentales, elle ne restera pas non plus sans effet sur la manière d'envisager les rapports du sujet à la morale et à l'éthique dans la mesure où le sujet, même dans ce 
qu'il peut avoir de proprement humain, vient nécessairement s'inscrire et se réaliser dans le monde social. Ses investissements et ses choix moraux et éthiques sont eux aussi inscrits dans l'historicité, ils ne pourront plus s'expliquer seulement par un rapport à une transcendance, mais prendront leur sens au sein même de ces tensions qui fondent ce que Touraine a appelé les deux faces de l'identité. La sphère de l'éthique ne peut plus être conçue comme un ensemble de valeurs préexistantes à la vie d'une société, elle est à la fois inscrite dans la production de la société et en tension avec elle. On pourrait dire aussi qu'elle prend son sens dans les failles qui existent entre les trois modes de socialisation que nous avons évoqués : intégration des normes, apprentissage des stratégies et réalisation de soi par rapport à un sujet historique. C'est au cœur même de ces divers modes de socialisation qu'un sujet moral peut apparaître.

Examinons donc l'impact de l'historicité sur la morale et sur l'éthique. Il apparaît tout d'abord et de manière simple que, en tant qu'acteur social, le sujet humain ne peut être le point de départ de l'éthique. Il surgit dans un monde moral déjà institué, fait de valeurs, de lois et de normes qui ont acquis une vie propre, relativement autonome et qui surdétermine les situations concrètes dans lesquelles chacun sera amené à faire des choix. Le sujet humain a dès lors affaire, de manière incontournable, avec des prescriptions que l'on peut considérer comme la résultante de I'histoire de I'humanité. Ce que Ricœur appelle « la face de nuit de l'éthique » (Ricœur, 1974 : 9), c'est-à-dire l'ensemble des normes, des lois, constitue effectivement une limite aux désirs, impose, consciemment ou inconsciemment à l'acteur certaines règles et certaines manières d'être qui guideront ses pratiques. On dira alors qu'il a appris certaines règles morales ou qu'il a été socialisé de telle ou telle manière. On pourra analyser et interpréter ses conduites à partir du poids des institutions qui pèse sur lui. Mais, ce faisant, on aboutira à une vision réductrice qui, d'une manière ou d'une autre, établira une chaîne causale simple entre les situations et les actions. On verra l'action comme une résultante des seules situations.

Or, une analyse des structures de l'action montre que l'action humaine, à la différence de l'action physique, " est médiation entre une situation et une visée " (Ladrière, 1981 : 224). La visée, c'est une orientation qui transforme la situation, non pas de manière arbitraire, mais en vue d'une réalisation de soi, grâce à une auto-implication de l'agent. Elle amène à concevoir chaque trajectoire comme une destinée irréductible et nécessairement unique tout en étant inscrite dans un monde social.

Ce caractère à la fois unique et social de la visée, de l'éthique pourrions-nous dire, ne peut être appréhendé à partir de la seule 
observation du chercheur. II ne peut apparaître qu'à travers un processus actif, qu'à travers une interaction entre le chercheur et le sujetacteur. C'est la raison pour laquelle nous pensons que l'approche biographique est susceptible de faire émerger les moments de tension entre les situations et les visées, moments producteurs des conduites qui organisent la trajectoire de chacun.

Nous voudrions donc montrer en quoi une telle conception nous fait avancer par rapport à notre problématique de départ. En quoi le récit de vie peut-il donner au chercheur des informations sur la manière dont les sujets-acteurs se situent par rapport aux règles ? Ce faisant, nous verrons comme la question elle-même subit une sorte de décentration, en ce sens qu'une forme de savoir n'apparaît au chercheur que si l'interviewé s'approprie ce savoir en même temps qu'il le communique.

II apparaît tout d'abord que le récit en tant que production peut être considéré comme un acte qui a pour effet de refigurer l'expérience temporelle confuse de l'agir humain ${ }^{9}$. À travers les histoires racontées sous forme d'intrigues qui se déroulent dans le temps, apparaît une forme d'intelligibilité complexe. Des liens logiques s'établissent entre l'intention et l'action, entre le motif et le projet, liens qui sont d'une autre nature que ceux qui s'établissent entre une cause et ses effets ${ }^{10}$. Et ce mode particulier d'inscription du sujet dans la temporalité est une manière de dire la capacité d'initiative du sujet. Seul un acteur est capable de produire un récit et c'est donc en tant qu'il est acteur que nous sommes amenés à l'écouter et à le comprendre. Et ceci a pour corollaire le fait que « dire le récit de sa vie fait acte pour le sujet »(Cockx et al., s.d.). Le récit ne peut donc être considéré par le chercheur comme un simple moyen d'information, il doit être compris comme un acte par lequel le sujet peut commencer quelque chose en racontant sa vie. Et cela a des conséquences importantes sur la relation entre le chercheur et celui qui parle, non seulement sur le plan du savoir produit, mais aussi pour celui qui s'exprime. Nous les évoquons brièvement pour conclure cet article.

F. Ferrarotti définit le récit biographique comme " une action sociale par laquelle un individu retotalise synthétiquement sa vie (la biographie) et l'interaction sociale en cours (l'interview) au moyen d'un récit interaction " (Ferrarotti, $1979: 143$ ). Cette interaction a donc un effet sur le narrateur qui, au cours de la narration elle-même, est amené à se poser des questions, à devenir alors partie prenante du processus de recherche enclenché par le chercheur. II entre alors dans ce que nous pourrions appeler un processus de formation, ou de transformation, et le fait de raconter sa vie va interférer avec la gestion même de cette vie. Ainsi, l'interaction entre le chercheur et ce qui était au départ son " objet " de recherche - c'est-à-dire pour 
nous le sujet dans sa manière de se situer par rapport aux règles -, cette interaction elle-même doit être considérée comme un moment de la vie du sujet en relation avec le chercheur. L'idéal d'une connaissance " objective " et comparative telle qu'elle pourrait apparaître à travers des questionnaires ou des entretiens plus ou moins directifs s'évanouit et est remplacée par une connaissance plus riche et plus complexe qui tient compte de la situation d'interaction et l'envisage comme une relation clinique.

Certes, cette relation clinique n'a pas de fonction thérapeutique et ne doit pas en avoir sous peine de dévier de sa finalité qui reste de produire des connaissances. Elle peut cependant, du fait de l'activité qui est mise en route par l'informateur, comporter un aspect de " formation " qui pourrait lui-même être intégré dans une recherche dont la nature et les finalités devraient être revues. Ceci reviendrait à opérer le passage vers une démarche de recherche qui serait également "éducative " au sens où elle permettrait une reprise par le sujet de ce qu'il est ou de ce qu'il est devenu à la suite d'événements constitutifs de son histoire.

Dans une telle perspective, le récit de vie permet au sujet qui réfléchit sur lui-même d'inventer des solutions morales nouvelles aux situations vécues et ainsi de s'autonomiser par rapport aux règles existantes. II introduit un autre rapport aux règles qui peut se construire à partir $d^{\prime}$ 'une mise en évidence des différents types de capitaux dont dispose le sujet ${ }^{11}$. Dans le domaine de la délinquance, on peut penser qu'une telle approche, que l'on pourrait qualifier de socioclinique, serait susceptible d'intervenir dans la formation des jugements moraux et dans l'analyse de leurs rapports avec les règles sociales et les lois existant dans la société. Elle permettrait peut-être d'envisager, dans une perspective dynamique, les aspects contraignants qui réduisent les possibilités d'action des sujets, contraintes externes et internes, mais aussi les éléments d'ouverture susceptibles d'être exploités.

On le voit, une telle manière d'envisager la question des rapports aux règles nous entraîne assez loin de la recherche centrée sur une étude objective et causale. Elle nous oblige à nous situer comme chercheur intervenant dans une situation que, par le moyen de l'entretien biographique, on vise à analyser. Elle nous place en quelque sorte sur un terrain plus clinique, rejoignant en cela les prescriptions de Touraine qui, d'une manière certes un peu différente, recommandait aussi que la sociologie devienne plus interventionniste et plus "clinique " (Dubet, $1990: 79$ ). 


\section{Notes}

1 II s'agit d'une recherche menée en vue de la présentation d'une thèse de doctorat en criminologie (Université catholique de Louvain) et qui a fait l'objet d'une publication intitulée Éthique et délinquance. La délinquance comme gestion de sa vie (Digneffe, 1989-a).

${ }^{2} \mathrm{Ce}$ point précis, ainsi que les conséquences que l'on peut en tirer, a fait l'objet d'un article intitulé Les présupposés idéologiques et théoriques des recherches relatives aux valeurs (Digneffe, 1989-b).

${ }^{3}$ Le point de départ de ce travail fut la parution de la thèse de doctorat de Kohlberg (1958), qui a été suivie d'une multitude de recherches. Travaillant à Harvard, il avait en effet obtenu des fonds privés qui lui permirent de mettre sur pied un centre d'études du développement moral qui attira bientôt de nombreux spécialistes en pédagogie, en psychologie génétique et en psychologie de la personnalité (Moessinger, 1989 : 43).

${ }^{4}$ Cette question a déjà été envisagée dans un article paru antérieurement (Digneffe, 1986).

${ }^{5}$ Par morale, on entendra ici l'ensemble des règles établies dans une société ou dans un groupe et qui forme un tout " institué ". Par éthique, on entendra plutôt le rapport que le sujet entretient avec ce qui lui apparaît comme valeur centrale, ou comme valeur première, et à partir de quoi il se positionnera par rapport aux règles morales et sociales en choisissant de les respecter ou de les transgresser.

${ }^{6}$ On se souvient que, au XIXe siècle, la criminologie s'est construite autour des recherches du psychiatre italien C. Lombroso. Celui-ci et les criminologues qui I'ont suivi s'efforçaient de proposer la mise en place d'un droit pénal « scientifique »qui tiendrait compte des déterminismes à la base des comportements humains et qui remplacerait le droit pénal " classique » qui trouve son origine dans la pensée de Beccaria et est au contraire fondé sur le postulat du libre arbitre.

${ }^{7}$ Les éléments repris dans cette partie ont fait l'objet de communications au cours d'un colloque qui s'est tenu à Louvain-la-Neuve du 27 au 30 septembre 1989 , sur le thème "Acteur social et délinquance. Une grille de lecture du système de justice pénale ». Les actes de ce colloque ont été publiés (Debuyst, 1990).

${ }^{8}$ Ces questions ont déjà été envisagées dans Digneffe, 1990 : 351-374.

${ }^{9}$ Nous nous référons ici aux analyses faites par P. Ricœur dans le volume I de Temps et récit (1982).

${ }^{10}$ À la suite de Ricœur (1977), nous utilisons ici le mot de cause dans le sens que lui a donné Hume : "pour celui-ci, la relation entre cause et effet implique que les antécédents et les conséquents sont logiquement indépendants, c'est-à-dire susceptibles d'être identifiés séparément (si une allumette met le feu à un explosif, je peux décrire l'allumette sans décrire l'explosif. II $n^{\prime} y$ a donc pas de lien logique entre la cause et l'effet. Or tel n'est pas le cas entre intention et action, entre motif et projet. Je ne peux pas identifier un projet sans parler de l'action que je ferai : il y a là un lien logique et non causal » (p. 134).

${ }^{11}$ Des pratiques de récit de vie-intervention commencent ainsi à se développer. Dans le cadre d'interventions réalisées dans un centre d'accueil pour toxicomanes, $\mathrm{F}$. Loicq décrit de manière concrète les terrains qui sont explorés par le récit de vie appelé alors « socio-analytique » (Loicq, 1987). De telles 
pratiques peuvent amener les sujets à analyser leur rapport à la toxicomanie et à la loi et à se repositionner éventuellement de manière différente. Ces recherches se rapprochent de celles menées par V. de Gaulejac et décrites dans La névrose de classe (1987).

\section{Références bibliographiques}

Boudon, R. et F. Bourricaud (1983). Dictionnaire critique de la sociologie. Paris : P.U.F.

Cockx, B., D. Gallez et G. de Villers (s.d.). Récit de vie et formation, in "Pratiques du récit de vie et théories de la formation ", Cahiers de la section des sciences de l'éducation, pratiques et théories, $\mathrm{n}^{\circ} 44$, Université de Genève, 53-71.

Cohen, A.K. (1955). Delinquent Boys. Glencoe, III. : Free Press.

Deburst, Ch. $(1985$, a.). Modèle éthologique et criminologie. Bruxelles : Mardaga.

DeBuYST, Ch. $(1985$, b.). " Jugement moral et délinquance. Les diverses théories et leur opérationnalisation. Kohlberg - ses études comparatives ", Déviance et société, vol. IX, $\mathrm{n}^{\circ} 2$ : 119-132.

Debuyst, Ch. (1990). Acteur social et délinquance. Une grille de lecture du système de justice pénale. Bruxelles : Mardaga.

Digneffe, Fr. (1986). "Morale de justice ou morale de responsabilité. Débat entre L. Kohlberg et $\mathrm{C}$. Gilligan à propos du développement du jugement moral ", Déviance et société, vol. X, $\mathrm{n}^{\circ} 1: 21-38$.

DiGneFFE, Fr. (1989-a.). Éthique et délinquance. La délinquance comme gestion de sa vie. Genève : Médecine et Hygiène, Méridiens-Klinscksieck.

DıGNEFFE, Fr. (1989-b.). Les présupposés idéologiques et théoriques des recherches relatives aux valeurs. Vaucresson : Séminaire international sur les normes et les valeurs, p. 15-24.

DignefFe, Fr. (1990). "L'acteur social et le sens de son utilisation dans les théories criminologiques, dans Ch. Debuyst ", Acteur social et délinquance. Une grille de lecture du système de justice pénale. Bruxelles : Mardaga, p. 251-274.

DUBET, Fr. (1990). "Socialisation des acteurs et sujet social ", dans Debuyst Ch., Acteur social et délinquance. Une grille de lecture du système de justice pénale. Bruxelles : Mardaga, p. 55-79.

Durkheim, E. (1967, $1^{\text {re }}$ éd. 1893). De la division du travail social. Paris : Alcan.

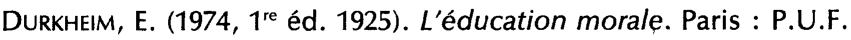

GILligan, C. (1977). "In a different voice : woman's conceptions of self and morality ", Harvard Educational Review, vol. 47, n 4 : 481-517.

Gillıgan, C. (1982). In a different voice. Cambridge, Mass. : Harvard Univ. Press.

HAWKINS, M.J. (1977). "A reexamination of Durkheim's theory of human nature ", Sociological Review, 25, (2) : 229-252.

HIRSCHI, T. (1969). Causes of Delinquency. Berkeley : University of California Press. 
JURKovic, G. et N.M. Prentice (1980). "The juvenile delinquent as a moral philosopher ", Psychological Bulletin, vol. 88, n 3 : 709-727.

KOHLBERG, L. (1958). The development of modes of moral thinking and choice in the years ten to sixteen, unpublished doctoral dissertation. Chicago : University of Chicago.

KoHlberG, L. et C. Gilligan (1971). "The adolescent as a philosopher : the discovery of the self in a postconventional world ", Daedalus, 1051-1086.

LADRIÈRE, J. (1981). "Éthique et nature ", dans Cl. BRUAIRE, La morale, sagesse et salut. Paris : Fayard, p. 221-247.

LADRIÈRE, J. (1984). "Droit naturel, droit, éthique ", Revue interdisciplinaire d'études juridiques, $\mathrm{n}^{\circ} 13: 90-97$.

LoICQ, F. (1987). "Le récit de vie centré sur l'héritage familial et la trajectoire sociale ", Cahiers de la dépendance, $n^{\circ} 7$.

MOESSINGer, P. (1989). La psychologie morale. Paris : P.U.F., coll. Que sais-je ?

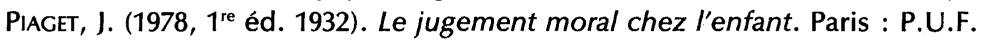

PIAGET, J. (1965). Études sociologiques. Genève : Droz.

RICøur, P. (1977). "Expliquer et comprendre ", Revue philosophique de Louvain, 126-147; repris dans RIçuR, Du texte à l'action, essais d'herméneutique, 2, Paris : Seuil, p. 161-182.

Ricœur, P. (1982, 1983, 1984). Temps et récit. Paris : Seuil, 3 volumes publiés.

SCHOTTE, J. (1990). "Vers une théorie du sujet ", dans Ch. DeBuYST, Acteur social et délinquance. Une grille de lecture du système de justice pénale. Bruxelles : Mardaga, p. 131-142.

Touraine, A. (1984). Le retour de l'acteur. Paris : Fayard. 